\title{
Analysis of Conformal Reflectarray on Cylindrical Surface
}

\author{
Yan $\mathrm{Qu}^{1,2}$, Wencan Peng ${ }^{1}$, Chenjiang Guo ${ }^{1}$ and Jun Ding ${ }^{1}$ \\ ${ }^{1}$ School of Electronics and Information, Northwestern Polytechnical University, Xi'an \\ 710129, China \\ ${ }^{2}$ CAST - Xi'an Institute of Space Radio Technology (CAST Xi'an), 710000, China \\ quyan0908@gmail.com
}

\begin{abstract}
As the important case of conformal arrays, conformal arrays on cylindrical surfaces have been widely used in radar and communication systems. This paper explores the chances of designing conformal reflectarray on cylindrical platform. An extended array theory method is proposed in this paper for analyzing the radiation patterns of cylindrical conformal reflectarray. The radiation characteristics of conformal reflectarray on convex and concave cylindrical surfaces are studied, and the performances of the two cases are compared with planar cases. It is shown that the cylindrical conformal reflectarray can be a good candidate of high gain antennas for applications where a slightly curved conformal platform is available.
\end{abstract}

Keywords: reflectarray antennas, array theory, conformal arrays, cylindrical surface

\section{Introduction}

As reflectarray antenna combines the advantages of both phased array and parabolic reflectors, which create a low mass and low cost high gain antenna, it has been received notable attentions in the last decade $[1,2]$. The surface of the reflectarray antenna consists of thousands of passive phased elements which are adjusted to create the collimated or shaped beam. Considering the large number of elements on the surface of the reflectarray antenna, the efficient full-wave analysis of the reflectarray is still quite challenging and various methods have been developed to calculate the far-field radiation pattern of the reflectarray antenna $[3,10]$.

As an important application of array antennas, conformal arrays have been widely used in such platform which can be some part of an airplane, train or other vehicle. The design that the conformal arrays become integrated with the platform causes fewer disturbances such as extra drag, and the electrical advantages of conformal array are typically wide angle scanning capabilities [4].

As the important case of conformal arrays, conformal arrays on cylindrical surface have been widely used in radar and communication systems. At first, the chance of designing conformal reflectarray on cylindrical platform is explored in this paper. An extended array theory method is proposed in this paper for analyzing the radiation patterns of cylindrical conformal reflectarray. The radiation performances of conformal reflectarray on concave and convex cylindrical surface are analyzed using method, and are compared with that of planar cases. It is demonstrated that cylindrical conformal reflectarray antennas have very similar performance with planar designs, which makes a good candidate of high gain antennas for applications where a slightly curved conformal platform is required.

\section{Model of the Radiation Characteristics of the Conformal Reflectarray}




\subsection{Derivation of Aperture Efficiency}

The radiation patterns of a planar reflectarray antenna with thousands of elements can be calculated quite efficiently through the traditional array theory method with proper element excitation $[5,10]$. In recent researches, it was demonstrated that the main beam direction, beam width and side lobe level calculated by this method show a good agreement with that calculated through time consuming full-wave simulation [3]. Therefore, the method based on the array theory is introduced to calculate the radiation patterns of conformal reflectarray. The expression of the array theory method, developed for planar reflectarray [3, 10], is generalized to analyze the radiation performance of conformal reflectarray in this paper. It has been demonstrated that the prerequisite that the array theory formulation can provide accurate results is that the elements on the reflectarray aperture are correctly modeled with mutual coupling included. Therefore, one of the key points of analyzing radiation patterns of conformal reflectarray through array theory method is the accurate modeling of the radiation characteristics of elements on the conformal surfaces. First of all, one should note that the peak of the element pattern is perpendicular to the local surface the element located on, and will not point in the same direction for a conformal reflectarray [6]. Furthermore, it is known that reflectarray antenna is working in the reflecting mode, which is different from phased array. Analysis of scattering features of conformal surfaces is more difficult than that of planar surfaces, and high order diffraction effects should be considered in general. Nonetheless, if the conformal surface is slightly curved, edge diffraction is minimized, then geometric optic method is still available for the analysis.

The radiation patterns of a conformal reflectarray with $\mathrm{M} \times \mathrm{N}$ elements can be calculated by the following general expression:

$$
\begin{aligned}
& E(\hat{u})=\sum_{m=1}^{M} \sum_{n=1}^{N} A_{m n}(\hat{u}) I\left(\vec{r}_{m n}\right) \\
& \hat{u}=\hat{x} \sin \theta \cos \phi+\hat{y} \sin \theta \sin \phi+\hat{z} \cos \theta
\end{aligned}
$$

where $A_{m n}(\hat{u})$ is pattern function of the element, $I\left(\vec{r}_{m n}\right)$ is excitation function of the element, $\vec{r}_{m n}$ is the position vector of the mnth element. The configuration of the conformal reflectarray is shown in Figure 1.

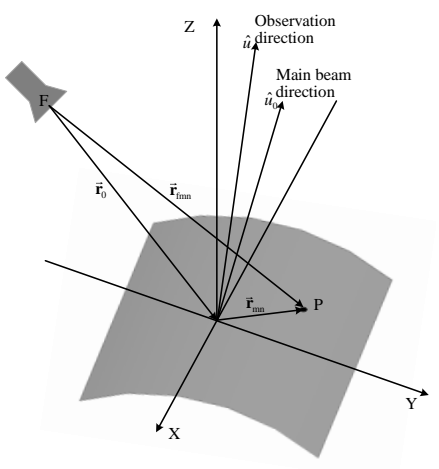

\section{Figure 1. The Configuration of the Conformal Reflectarray}

As the conformal reflectarray surface discussed in this paper is slightly curved, each cell can be approximated with a planar surface that is tangential to the conformal surface. Then, the pattern function of element can be approximated with model with its peak normal to the cell surface [7]. The exact incidence angle of each element and the orientation of the local cell coordinates are two important considerations for 
element pattern function model. The first one controls the receiving mode element pattern, and the second one controls the transmitting mode element pattern.

The element pattern function under transmitting mode can be written as

$$
A_{m n}(\theta, \phi) \approx \cos ^{\mathrm{q}_{\mathrm{e}}}\left(\theta_{\text {local }}\right) e^{j k\left(\bar{r}_{m n}[\hat{u})\right.}
$$

In comparison with the expression of planar design, it is worth noting that the effects of surface curvature on the transmitting mode element pattern are taken into account through using of local element coordinates for each element.

The excitation function of element is determined by the incident field of feed horn and the scatter property of element. The incident field on the conformal surface can be obtained by modeling the feed horn pattern using function and taking into account the energy decay and phase delay associated with the distance between the feed horn and the element, it can be written as

$$
E_{\text {incmn }}=\frac{\cos ^{\mathrm{q}_{\mathrm{f}}}\left(\theta_{\mathrm{fmn}}\right)}{\left|\vec{r}_{\mathrm{fmn}}\right|} e^{-j k\left|\overrightarrow{\widetilde{f r m}}_{\mathrm{fm}}\right|}
$$

Where $\theta_{\mathrm{fmn}}$ the spherical angle of the mnth element is in the feed coordinate $\left|\vec{r}_{\mathrm{fmn}}\right|$ is the distance between the feed horn and the mnth element.

The scattering property of element can be represented by the receiving mode element pattern which determines the reflect energy from element based on the incidence angle and pattern shape of element, that is

$$
\Gamma_{\mathrm{mn}}=\cos ^{\mathrm{q}_{\mathrm{e}}}\left(\theta_{\mathrm{emn}}\right) \mathrm{e}^{\mathrm{j} \phi_{\mathrm{mn}}}
$$

While the expression here is similar to the planar design, the difference is that for conformal reflectarray the element angle $\theta_{\mathrm{emn}}$ depends not only on the element position, but also on the orientation of local cell surface. $\phi_{\mathrm{mn}}$ is required phase delay of the mnth element to set the main beam in the $\hat{u}_{0}$ direction.

With these approximations and expansions, the radiation pattern of a conformal reflectarray antenna can be written as

$$
\begin{aligned}
E(\theta, \varphi)= & \sum_{m=1}^{M} \sum_{n=1}^{N} \cos ^{\mathrm{q}_{\mathrm{e}}}\left(\theta_{\text {local }}\right) \frac{\cos ^{\mathrm{q}_{\mathrm{f}}}\left(\theta_{\mathrm{fmn}}\right)}{\left|\overrightarrow{\mathrm{r}}_{\mathrm{f}}\right|} \\
& \times \cos ^{\mathrm{q}_{\mathrm{e}}}\left(\theta_{\text {emn }}\right) \mathrm{e}^{\mathrm{j} \phi_{\mathrm{mn}}} e^{-j k\left(\left|\mathrm{r}_{\mathrm{f}}\right|-\vec{r}_{m n} \hat{\imath}\right)}
\end{aligned}
$$

This expression is obtained by the expanded array summation method without consideration of cross-polarization. If the cross-polarization of reflectarray is concerned, the polarization of feed horn and elements need to be accounted $[3,10]$.

\subsection{Gain Calculation}

For calculating the gain of reflectarray antenna, it is necessary to determine the aperture efficiency of the reflectarray antenna. Among many kinds of efficiency factors considered in conventional reflector antenna, two major terms that influence the reflectarray antenna gain are the spillover and taper efficiency [10].

The expression (5) for calculating radiation pattern has accurately taken the illumination on the conformal surface into account. In other words, it is meaning that the effect of taper efficiency has already been considered in that expression. Therefore, the spillover efficiency is left to pay attention. As we known, the definition of the spillover efficiency is that the percentage of radiated power from the feed that is intercepted by the reflecting surface. The evaluation of that can be written as 


$$
\eta_{s}=\frac{\iint_{\sigma} \vec{P}(\vec{r}) \sqsubset d \vec{s}}{\iint_{\Sigma} \vec{P}(\vec{r}) \square d \vec{s}}
$$

Where both integrals are fluxes of the Poynting vector $\vec{P}(\vec{r})$ through certain surface areas. The denominator is the total power radiated by the feed; therefore, the integral is performed over the entire surface area of a sphere centered at the feed, denoted by $\Sigma$. The numerator is the power incident on the array aperture, thus it is evaluated over a portion $\sigma$ of the sphere. As the portion $\sigma$ and the array aperture share the same solid angle with respect to the feed, the numerator can be computed through the conformal surface numerically. This is generally the same approach one follows for spillover efficiency calculations of a planar reflectarray antenna, however, the difference here is that the integration has to be performed over a curved surface. The gain of the reflectarray antenna is then computed using

$$
G=\eta_{s} D_{\max }
$$

Where $D_{\max }$ is the maximum directivity of the reflectarray antenna, which is calculated using expression (5). It should be noted that the calculations of the gain discussed here don't include the factors associated with the feed loss, the element loss, and polarization loss. In most cases for reflectarray antenna with low-loss substrates, these factors don't contribute much to the overall loss of the reflectarray antennas [11].

\section{Numerical Results}

\subsection{Radiation Patterns of Conformal Reflectarray on the Cylindrical Surface}

In this section, the radiation characteristics of reflectarray on conformal sector cylindrical surface are studied for providing an insight into many radiation characteristics of conformal reflectarray antennas. The cross-section of the conformal reflectarray antenna is shown in Figure 2, where concave and convex cylindrical surfaces are compared with the planar design with the same surface area. In this figure, $R_{c}$ denotes the radius of the cylinder for the conformal surface, and D is the physical aperture size.

It should been noted that for conformal arrays with broadside beam, large element space (relative to wavelength) will result in generation of grating lobes in visible range, however, this error can be substantially minimized for the element spacing in the order of halfwavelength [7]. Therefore, this value is chosen for all numerical studies in this paper. In addition, it is important to point out that there is a direct relation between the element spacing and surface curvature, and for the half-wavelength element spacing used in this paper, the maximum surface curvature of a single element is in the order of 1/20th of a radian, which ensures that a planar multifaceted approximation for the element performance is a suitable assumption. 


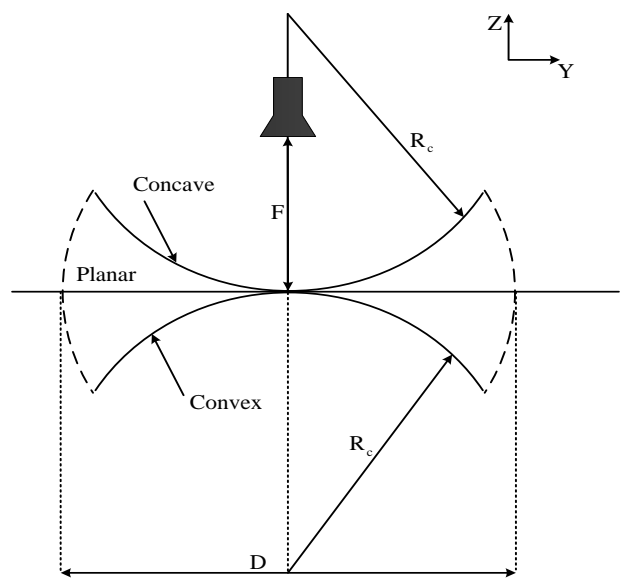

Figure 2. Cross-section of the Conformal Cylindrical Reflectarray Antenna

The numerical method presented in this paper is applied to analyze the radiation pattern of sector reflectarray antenna mounted on cylindrical surfaces. For a meaningful comparison of the surface curvature effect, the feed properties and the physical aperture size are kept constant, and the only parameter changed is the cylinder radius. The reflectarray antennas have a $20 \lambda \times 20 \lambda$ aperture area, and are designed to generate a broadside beam. Ideal phasing elements are used and the element pattern is modeled as a $\cos ^{1}$ function with its peak normal to the local surface and no azimuth dependence. The reflectarray configuration is prime focus with an $F / D=0.75$, and the power $q_{f}$ of the radiation pattern of feed horn is 6.5 at $12 \mathrm{GHz}$ varying linearly from 5 at $11 \mathrm{GHz}$ to 8 at $13 \mathrm{GHz}$.

The comparisons of radiation patterns between cylindrical reflectarray with various cylinder radiuses and planar reflectarray are shown in Figure3, where 3 (a) for radiation patterns of concave cases in xoz plane, 3 (b) for radiation patterns of convex cases in xoz plane, 3 (c) for radiation patterns of concave cases in yoz plane, and 3 (d) for radiation patterns of convex cases in yoz plane.

It can be seen from Figure 3 (a) and (b) that whether concave case or convex case, the radiation patterns of cylindrical reflectarray in the xoz plane are almost similar to the planar design except that the side lobe around $\pm 70^{\circ}$ is lower than planar design. It can be seen from Figure 3 (c) and (d) that for both concave case and convex case, the radiation patterns of cylindrical reflectarray in the yoz plane show a noticeable difference with planar design. In particular, grating lobes are observed at area of $|\theta| \geq 60^{\circ}$ for both conformal cases with $\mathrm{Rc}=10 \lambda$. It is mainly caused by fast phase changing of element located at aperture edge [7]. Compared with planar design, the concave cases show higher side lobe, on the contrary, the convex cases show lower side lobe; both cases show wider beam width. However, the radiation patterns of the conformal cases approach that of planar design as cylinder radius increases.

In order to further illustrate the effect of cylinder radius $\mathrm{Rc}$ on the main beam width and side lobe level of the radiation patterns of the cylindrical reflectarray, the normalized halfpower beam width (3dBBW) and peak side lobe level (PSLL) of radiation patterns in the yoz plane as a function of cylinder radius is given in Figure 4. It can be seen from figure 4 (a) that in comparison between the two conformal cases, the convex case shows much wider beam width. In general, both two cases show defocusing effects, that is, a wider beam width in comparison with a planar design. This is mainly caused by the fact that the elements on conformal surface radiate in the outward (convex) or inward (concave) radial directions of the 
cylinder. It should be noted that as the cylinder radius increases, the normalized 3dBBW of both conformal cases decrease and approach that of planar case. It can be observed that as the cylinder radius increases, the normalized PSLL of the concave case decreases from $5.8 \mathrm{~dB}$ to $0 \mathrm{~dB}$, but the convex case increases from $-5.6 \mathrm{~dB}$ to $0 \mathrm{~dB}$.

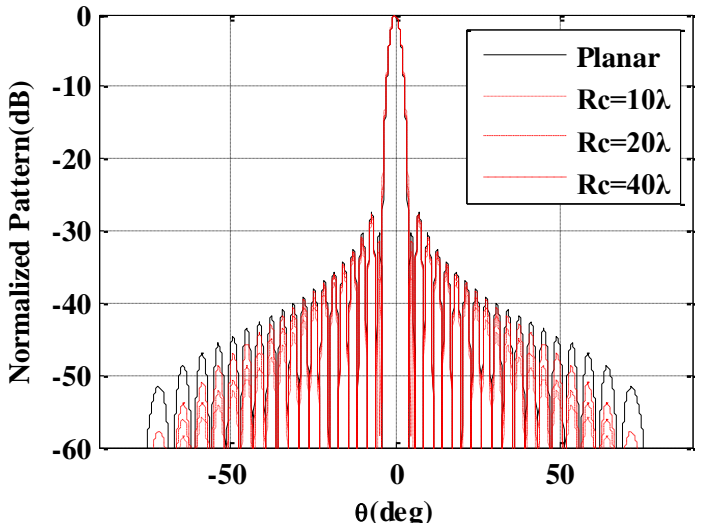

(a)

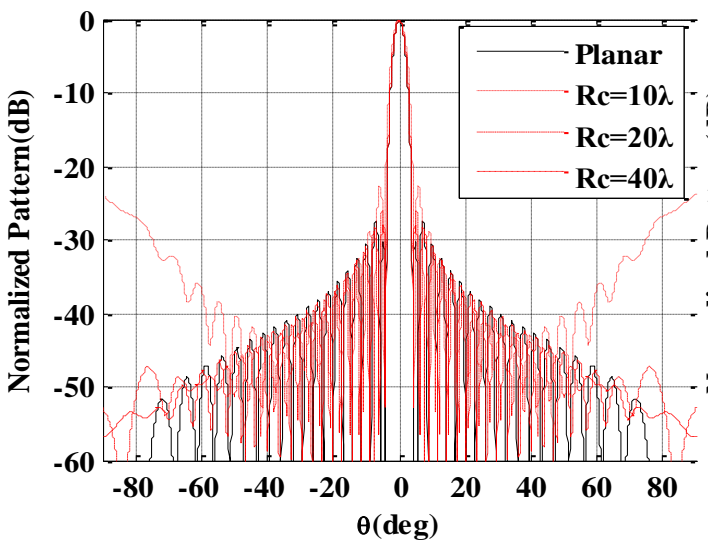

(c)

(b)

(d)
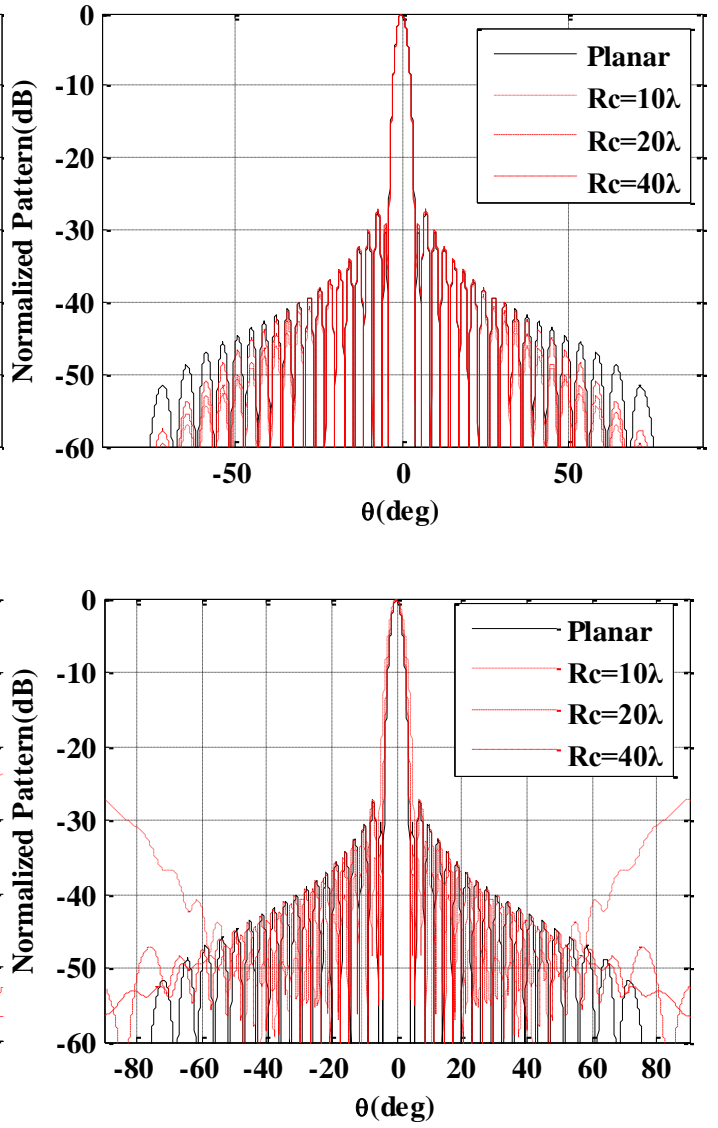

Figure 3. Radiation Patterns of Cylindrical Reflectarray

It is necessary to point out that for a cylindrical reflectarray with a given aperture size D, the cylinder radius cannot be smaller than D/3. Smaller cylinder radius will not be practical since: 1. the element will be in shadow region of feed, which results in that the element cannot be properly excited, 2. the pattern of elements at the sector edge point far away from the broadside, which results in defocusing effects and grating lobes.

The main title (on the first page) should begin $13 / 16$ inches (7 picas) from the top edge of the page, centered, and in Times New Roman 14-point, boldface type. Capitalize the first letter of nouns, pronouns, verbs, adjectives, and adverbs; do not capitalize articles, coordinate conjunctions, or prepositions (unless the title begins with such a word). Please initially capitalize only the first word in other titles, including section titles and first, second, and third-order headings (for example, "Titles and headings" - as in these guidelines). Leave two blank lines after the title. 


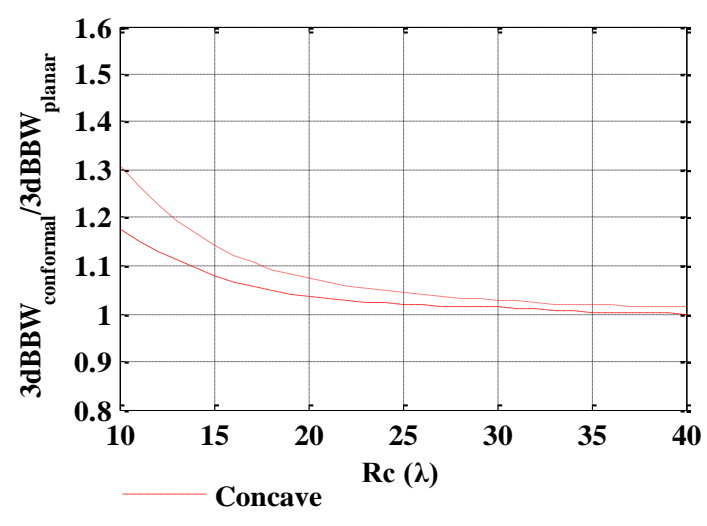

(a)

(b)

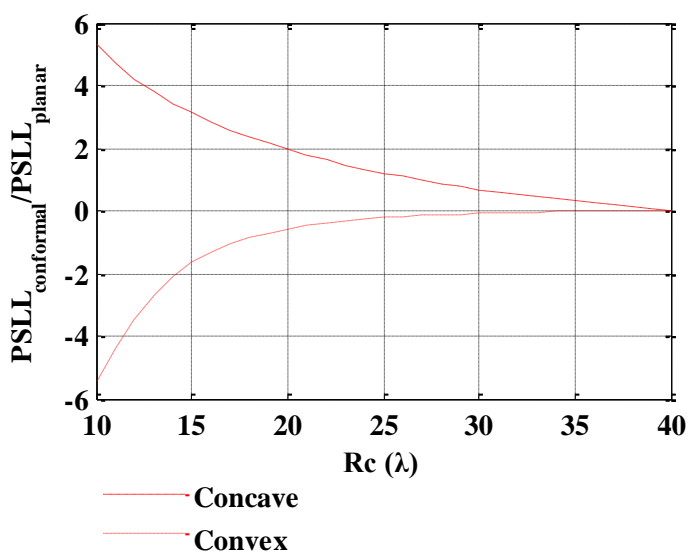

\section{Figure 4. Normalized 3dBBW and PSLL in the Yoz Plane as Function of Cylinder Radius}

\subsection{Gain and Bandwidth of Conformal Reflectarray on the Cylindrical Surface}

In order to illustrate the effect of cylinder radius on the gain of cylindrical reflectarray, the gain of cylindrical reflectarray as a function of cylinder radius is given in Figure 5. It can be observed that as the cylinder radius increases, the gain of both conformal cases increase, and approach the gain of planar design, which still keep a small reduction, but the concave cases always keep a little higher gain than the convex cases. As it is known that the reflectarray antenna gain is directly proportional to its projected aperture, for a fixed aperture size, the projected aperture of conformal reflectarray antennas will decease as the cylinder radius increases, which results in that phenomenon observed in Figure 5.

The bandwidth of the reflectarray antenna is affected by two main factors: one is the phase error associated with the spatial delay, and the other one is the bandwidth of elements $[2,9]$. While the bandwidth of element generally depends on the design scheme of element, to study the effect of cylinder radius on the bandwidth of the reflectarray antenna without consideration of element design scheme, we assume that the reflection phase of element is frequency-independent, that is, only the bandwidth limitation associated with spatial delay is taken in account. Figure 6 shows the normalized gain of a $20 \lambda \times 20 \lambda$ reflectarray antenna with planar and conformal aperture $(\mathrm{Rc}=20 \lambda)$ as a function of frequency.

It is worth noting that the bandwidth performance of both cases is a function of cylinder radius. These results are given in Figure7. For the convex cases, the bandwidth of cylindrical reflectarray increases monotonously with Rc. For the concave cases, the bandwidth of cylindrical reflectarray increases with Rc then decreases, which makes an optimum cylinder radius for the maximum bandwidth of the cases. When the cylinder radius reaches $30 \lambda$, the maximum bandwidth $20.8 \%$ is obtained. 
International Journal of Signal Processing, Image Processing and Pattern Recognition Vol.8, No.3 (2015)

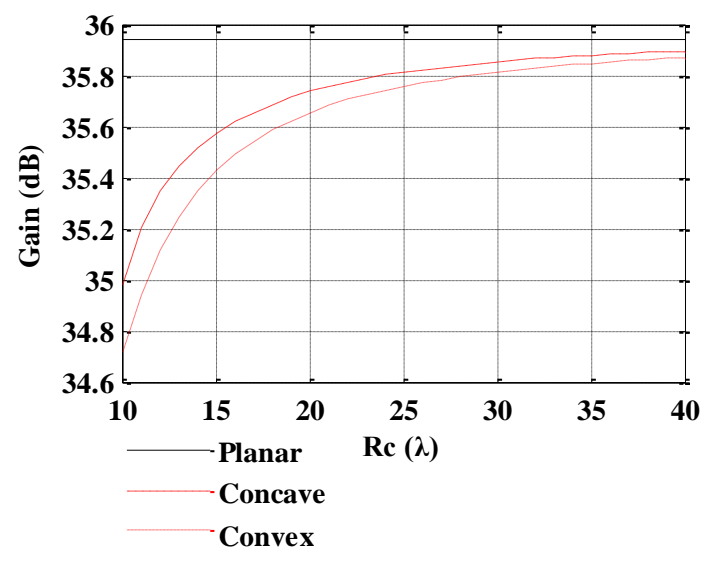

Figure 5. The Gain of Cylindrical Reflectarray as a Function of Cylinder Radius

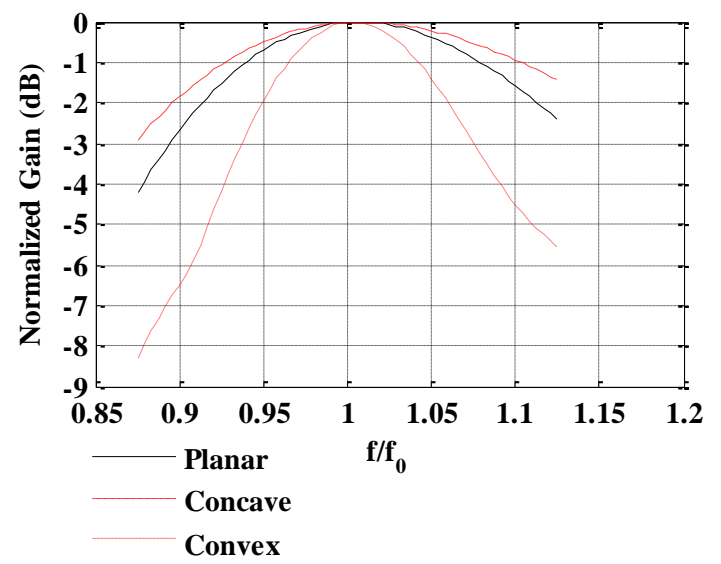

Figure 6. Normalized Gain as a Function of Frequency

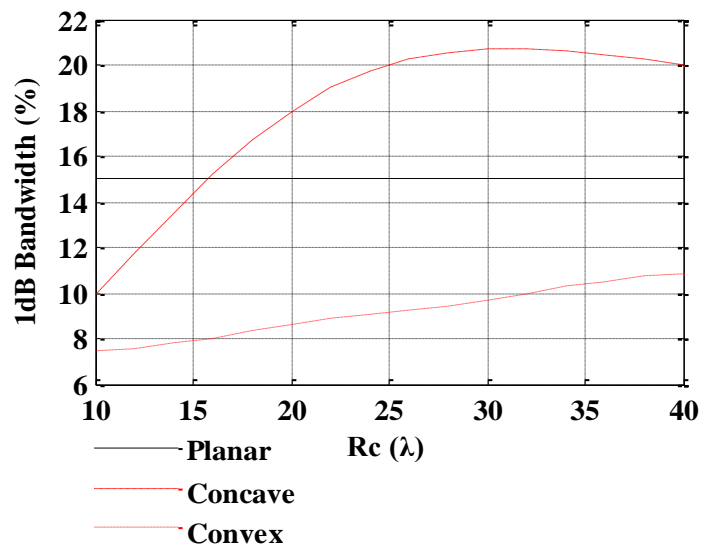

Figure 7. 1dB Bandwidth as a Function of Cylinder Radius

\section{Conclusion}

The possibility of designing conformal reflectarray antennas is explored numerically. An expanded array theory method is presented to calculate the radiation pattern of conformal reflectarray antennas. Then this method is utilized to analyze the radiation characteristics of 
concave and convex conformal reflectarray on cylindrical surfaces, and the performances of cylindrical conformal cases are compared with planar design. It is observed that both cylindrical conformal cases show wider beam width than planar design. The concave cases show higher PSLL, while the convex cases show lower PSLL. In addition, the bandwidth can be improved through a concave cylindrical conformal design. The investigations in this paper illustrate that for some high gain antenna application where a slight curved conformal platform is available, a conformal reflectarray antenna can be a suitable candidate for the design

\section{Acknowledgements}

This work is supported in part by the National Defense Pre-Research Foundation of China under Grant No.9140A01010412HK03004 and the Aerospace Innovation Fund under Grant No. HTCXJJKT-22.

\section{References}

[1] D. G. Berry, R. G. Malech, and W. A. Kennedy, "The reflectarray antenna. IEEE Transactions on Antennas and Propagation, vol. 6, no. 11, (1963).

[2] J. Huang and J. A. Encinar, "Reflectarray Antennas", J. Wiley \& Sons, Hoboken, NJ, (2008).

[3] P. Nayeri, A. Z. Elsherbeni and F. Yang, "Radiation analysis approaches for reflectarray antennas", IEEE Antennas and Propagation Magazine, vol. 1, no. 55, (2013).

[4] L. Josefsson and P. Persson, "Conformal Array Antenna Theory and Design", J. Wiley \& Sons, Hoboken, NJ (2006).

[5] J. Huang and R. J. Pogorzelski, "A ka-band microstrip reflectarray with elements having variable rotation angles", IEEE Transactions on Antennas and Propagation, vol. 5, no. 46, (1998).

[6] R. L. Haupt, “Antenna Array: A Computational Approach”, J. Wiley \& Sons, Hoboken, NJ (2010).

[7] R. J. Mailloux, "Phased Array Antenna Handbook $2^{\text {nd }}$ edition", Artech House, Boston, MA (2005).

[8] P. Nayeri, F. Yang and A. Z. Elsherbeni, "Radiation characteristics of conformal reflectarray antennas", Antennas and Propagation (APSURSI), 2011 IEEE International Symposium on. (2011), June 3-8; Spokane, WA.

[9] D. M. Pozar, "Bandwidth of reflectarrays", Electronics Letters, vol. 21, no. 39, (2003).

[10] Y. Qu, C. J. Guo, H. Guo and J. Ding, "Fast analysis for aperture efficiency and radiation patterns of reflectarray antennas", International Journal of Signal Processing, Image Processing and Pattern Recognition, vol. 4, no. 7, (2014).

[11] D. M. Pozar, S. D. Targonski, and H. D. Syrigos, "Design of millimeterwave microstrip reflectarray", IEEE Transactions on Antennas and Propagation, vol. 2, no. 45, (1997).

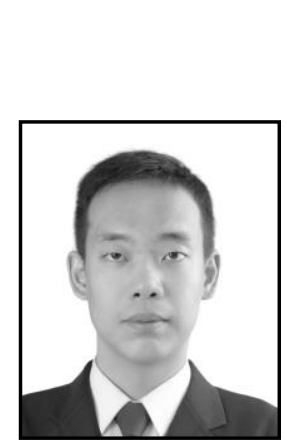

\section{Author}

Yan Qu, He obtained an MS in Electronic and Information Engineering from the school of Electronic and Information, Northwestern Polytechnic University, 2006. Then, he was recommended to a PhD in Electronic Science and Technology from the school of Electronic and Information, Northwestern Polytechnic University. His researches interesting are array synthesis, design of reflectarray. 
International Journal of Signal Processing, Image Processing and Pattern Recognition Vol.8, No.3 (2015) 